\title{
Energetics and Electronic Structures of Inclusion Compounds of Large Fullerenes and Cycloparaphenylenes
}

\author{
Yuya Nagasawa and Susumu Okada*
}

Graduate School of Pure and Applied Sciences, University of Tsukuba, 1-1-1 Tennodai, Tsukuba, Ibaraki 305-8571, Japan

The energetics and electronic structures of large fullerenes included within $[n]$ cycloparaphenylenes $([n] \mathrm{CPPs})$ are investigated using density functional theory. We observe that the large fullerenes included within the $[n] \mathrm{CPP}\left([n] \mathrm{CPP} \supset \mathrm{C}_{n}\right.$, where $n=76$ and 78) are energetically stable and the inclusion reactions are exothermic for the $[n]$ CPP with a diameter of $1.49 \mathrm{~nm}$ or larger. Owing to quantum confinement of the electronic states of the guest fullerene molecules by the host $[n] \mathrm{CPP},[n] \mathrm{CPP} \supset \mathrm{C}_{60}$ possess a staggered electronic structure near the energy gap between the highest occupied and the lowest unoccupied states, leading to a narrower energy gap than that of fullerenes and CPP. We also demonstrate that the electron states associated with the fullerene exhibit an upward shift upon inclusion owing to the quantum confinement effect on the $\pi$ states of fullerenes by the CPP.

\section{Introduction}

The electronic structures of nanoscale carbon materials depend on their size, local network geometry, dimensionality, and boundary condition. Nanocarbon materials consisting of only hexagonal rings exhibit a symmetric electronic structure in their $\pi$ electron states with respect to the Fermi level. Hydrocarbon molecules intrinsically possess a moderate energy gap between the highest occupied and the lowest unoccupied states, which basically decreases and asymptotically approaches zero with a larger $\pi$ electron network. ${ }^{1-3)}$ Graphene is the infinite version of an $\mathrm{sp}^{2}$ nanocarbon material, so it is a zero gap semiconductor with pairs of linear dispersion bands at the Fermi level. ${ }^{4-6)}$ By imposing boundary conditions on the hexagonal covalent networks of $\mathrm{C}$ atoms, $\mathrm{sp}^{2} \mathrm{C}$ atoms can form versatile nanoscale network materials, leading to the sponge, ${ }^{7-11)}$ tubular, ${ }^{12-15)}$ and hollow cage ${ }^{16-22)}$ structures. These nanoscale network materials exhibit interesting variations in their electronic structures near the Fermi

${ }^{*}$ E-mail address: sokada@comas.frsc.tsukuba.ac.jp 
level. A tubular form of $\mathrm{sp}^{2} \mathrm{C}$ atoms, carbon nanotubes, can exhibit electronic structures ranging from a semiconductor to metal depending on their chirality and diameter. ${ }^{13-15)}$ The electronic structures of fullerenes are sensitive to their cage size and network topology, ${ }^{18-22)}$ although their $\pi$ electron states can be characterized by a spherical harmonica owing to their approximately spherical distributions of electrons on the cages. ${ }^{21)}$

Condensed phases consisting of these nanocarbon materials are also fascinating materials that exhibit further variations in their physical properties which are differet from a simple extrapolation of the sum of each constituent. Fullerenes form close packed structures in their condensed phases, where the molecules are weakly or tightly bound to each other depending on the synthesis conditions. ${ }^{23-26)}$ Solid $\mathrm{C}_{60}$ can either be semiconductors or metals depending on the mutual molecular arrangements and intermolecular binding forms. Carbon nanotubes also form condensed phases, where the structures are characterized by a bundle of nanotubes owing to their tubular structure. ${ }^{27-29)}$ In the condensed phases, the bundles possess a tiny energy band gap owing to the weak intertube interactions, even though the bundles solely consist of metallic nanotubes. In addition to the homogeneous condensed phases of these nanocarbon materials, they can construct heterogeneous condensed structures by assembling appropriate constituent units, leading to hierarchical condensed phases with mixed dimensionality. Fullerenes can be encapsulated in the inner spacing of nanotubes, resulting in nanoscale peapods that exhibit unusual electronic structures. ${ }^{30-34)}$

Recently, interesting molecular complexes have been synthesized by mixing cyclohydrocarbon molecules and fullerenes. $\mathrm{C}_{60}$ is included in the inner spacings of cycloparaphenylene (CPP) and cyclochrysenylene as molecular inclusion complexes with a Saturn-like shape. ${ }^{35,36)}$ In addition to $\mathrm{C}_{60}, \mathrm{C}_{70}$ also forms similar complexes with CPP. ${ }^{37)}$ These facts imply that a further structural variation of such inclusion complexes is expected by choosing an appropriate combination of fullerene and cyclohydrocarbon molecules. The electronic structure variation of these constituent nanocarbon molecules makes the complexes exhibit unique electronic structures. Thus, in this paper, we aim to investigate the energetics and electronic structures of the inclusion complexes of large fullerenes, $\mathrm{C}_{76}{ }^{38)}$ and $\mathrm{C}_{78},{ }^{39,40)}$ and $[n] \mathrm{CPP}$, exhibiting unusual electronic structures, using density functional theory. Our calculations showed that the electronic structure of the inclusion complexes depends not only on the constituents but also on their mutual arrangements. We also found that the stability of the complexes is insensitive to the molecular species but is rather determined by the spacing between fullerene and CPP. 


\section{(a)}

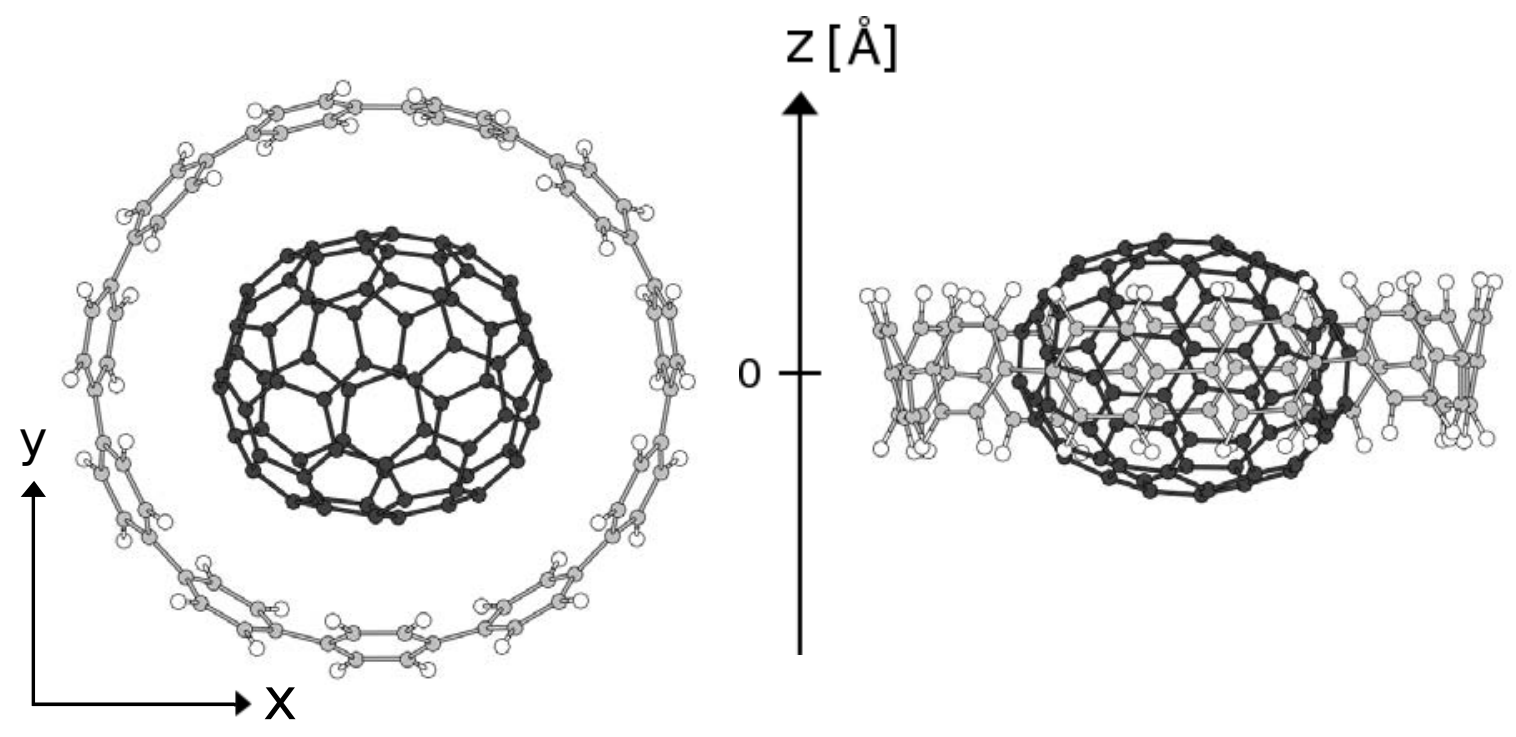

\section{(b)}
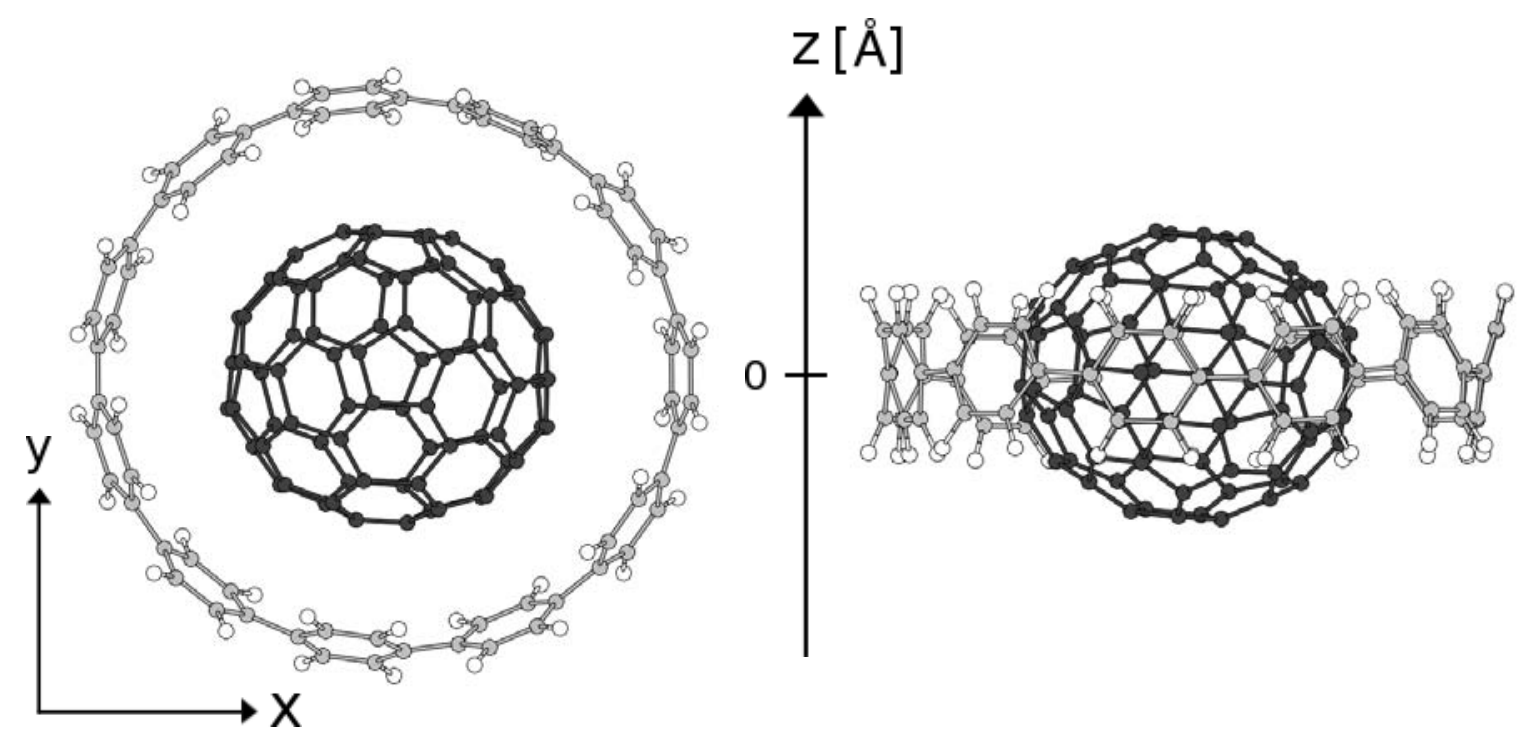

Fig. 1. Top and side views of optimized structures of (a) $[11] \mathrm{CPP} \supset \mathrm{C}_{76}\left(D_{2}\right)$ and (b) [11]CPP $\supset \mathrm{C}_{78}\left(C_{2 v}^{\prime}\right)$. The dark gray, light gray, and white circles denote $\mathrm{C}$ atoms belonging to fullerenes, $\mathrm{C}$ atoms belonging to [11]CPP, and $\mathrm{H}$ atoms, respectively. 


\section{Calculation methods and structural model}

All geometric and electronic structures of the inclusion complexes consisting of large fullerenes and CPP were conducted using density functional theory ${ }^{41,42)}$ implemented in the STATE program package. ${ }^{43)}$ For describing the exchange-correlation potential among the interacting electrons, we used the local density approximation, ${ }^{44,45)}$ which allowed us to undertake a qualitative discussion on the geometries and the energetics of the molecular complexes bound through weak inter unit interactions. We used ultrasoft pseudopotentials generated by the Vanderbilt scheme to describe the interaction between the valence electron and atomic ion containing nuclei and core electrons. ${ }^{46)}$ The valence wave function and deficit charge density were expanded by the plane wave basis set with cutoff energies of 25 and 225 Ry, respectively. To investigate the energetics and electronic structure of an isolated inclusion complex, we adopted the super cell of a large cubic cell with a length parameter of $41.57 \AA$. Brillouinzone integration was performed with the $\Gamma$ point sampling. The atomic structure was fully optimized until the force acting on each atom was less than $5 \mathrm{mRy} / \AA$. In this work, we focussed on two large fullerenes, $\mathrm{C}_{76}\left(D_{2 d}\right)$ and $\mathrm{C}_{78}\left(C_{2 v}^{\prime}\right)$, as the guest molecules for the $[n] \mathrm{CPP}$. These two fullerenes have been experimentally synthesized and extracted from soot. To investigate the effects of the interunit spacing on the electronic and geometric structures of the inclusion complexes, we considered [10]CPP, [11]CPP, and [12]CPP as the host molecule, which consist of 10,11, and 12 phenyl groups, respectively. These CPP have a staggered arrangement of phenyl groups as their stable conformations. In all complexes, fullerenes were located at the center of CPP. For estimating the inclusion energy of fullerenes in [11]CPP, we assume the reaction coordinate along the molecular axis of [11]CPP, which is normal to the CPP ring. During the calculation along the reaction coordinate, internal atomic coordinates are fixed, because the force acting on each atom is smaller than the force cutoff. The formation energy $\Delta E$ is also evaluated by $\Delta E=E_{\text {comp }}-E_{\text {full }}-E_{\mathrm{CPP}}$ where $E_{\text {comp }}, E_{\text {full }}$, and $E_{\mathrm{CPP}}$ are the total energies of inclusion complex, isolated fullerene, and [11]CPP, respectively.

\section{Result and discussion}

Figure 2 is the inclusion energy of fullerenes in CPP as a function of the diameter of CPP. The inclusion energy was evaluated by the reaction: fullerene $+[n] \mathrm{CPP} \rightarrow[n] \mathrm{CPP} \supset \mathrm{C}_{n}+\Delta E$. The inclusion energy was sensitive to the diameter of CPP but insensitive to the encapsulated fullerene species, because of the similar diameters of $\mathrm{C}_{76}$ and $\mathrm{C}_{78}$. The calculated inclusion energies were $0.32,-1.22,-0.78,0.19,-1.22$, and $-0.74 \mathrm{eV}$ for $[10] \mathrm{CPP} \supset \mathrm{C}_{76},[11] \mathrm{CPP} \supset \mathrm{C}_{76}$, $[12] \mathrm{CPP} \supset \mathrm{C}_{76},[10] \mathrm{CPP} \supset \mathrm{C}_{78},[11] \mathrm{CPP} \supset \mathrm{C}_{78}$, and [12]CPP $\supset \mathrm{C}_{78}$, respectively. For $\mathrm{C}_{76}$ and 


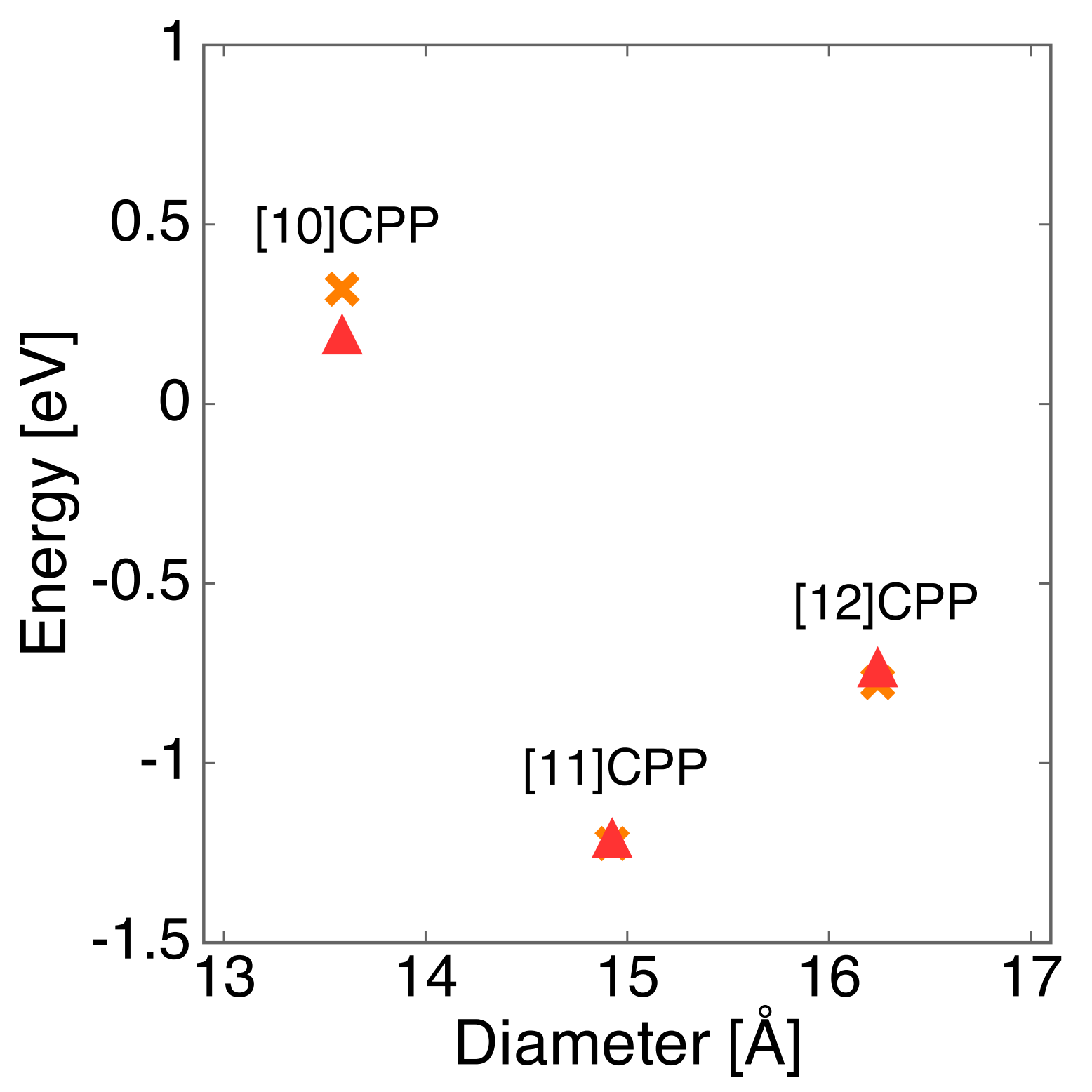

Fig. 2. The inclusion energy of fullerenes in $[n]$ CPP as a function of the diameter of CPP. Crosses and triangles denote the inclusion energy for $\mathrm{C}_{76}$ and $\mathrm{C}_{78}$, respectively.

$\mathrm{C}_{78}$, the inclusion reactions were exothermic for [11]CPP and [12]CPP and endothermic for [10]CPP. The inner space of [10]CPP was insufficient to accommodate these fullerenes. Under the inclusion structure, the space between the fullerenes and [10]CPP was $2.92 \AA$. In contrast, for the $[11] \mathrm{CPP}$, the interunit spacing was $3.14 \AA$, which led to the largest energy gain upon the inclusion of fullerene.

It is worth investigating the inclusion process of fullerene in [11]CPP for providing a theoretical insight into the reaction. Figure 3 shows the energy potential surfaces along the molecular axis of [11]CPP for $\mathrm{C}_{76}$ and $\mathrm{C}_{78}$ fullerenes. The potential surfaces monotonically 
Table I. The calculated inclusion energy of $\mathrm{C}_{76}$ and $\mathrm{C}_{78}$ fullerenes in $[n] \mathrm{CPP}$.

\begin{tabular}{lll}
\hline$[n] \mathrm{CPP}$ & $\mathrm{C}_{76}$ & $\mathrm{C}_{78}$ \\
\hline 10 & 0.32 & 0.19 \\
11 & -1.22 & -1.22 \\
12 & -0.78 & -0.74 \\
\hline
\end{tabular}

(a)

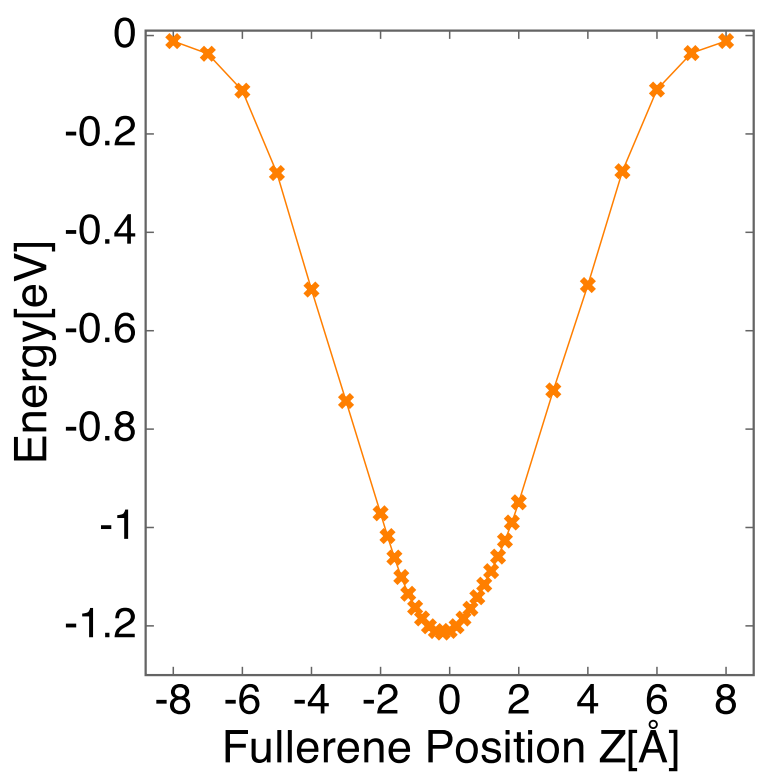

(b)

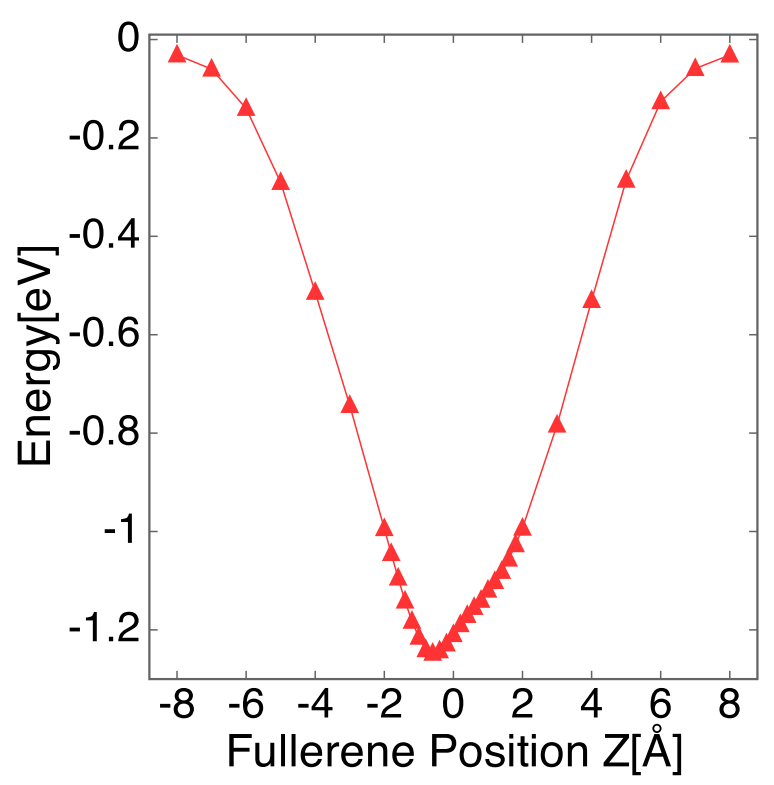

Fig. 3. The potential energy surfaces along the [11]CPP axis for (a) $\mathrm{C}_{76}$ and (b) $\mathrm{C}_{78}$.

decreased with approaching the inner spacing of [11]CPP. Thus, the inclusion reactions of $\mathrm{C}_{76}$ and $\mathrm{C}_{78}$ fullerenes in [11]CPP did not exhibit any reaction barriers, so that the inclusion complexes of the fullerenes and [11]CPP could be spontaneously synthesized by a simple mixing under the appropriate experimental conditions. By carefully checking the potential surface around the energy minima, the energy potential exhibited unusual features because of the asymmetric geometric structures of $\mathrm{C}_{76}$ and $\mathrm{C}_{78}$ fullerenes with respect to [11]CPP. The asymmetries cause a dislodgment of the energy minima from the center of mass of [11]CPP. The minima were dislodged from the center of [11]CPP by -0.2 and $-0.6 \AA$ along the reaction coordinate for $\mathrm{C}_{76}$ and $\mathrm{C}_{78}$ fullerenes, respectively. Note that the dislodgement was sensitive to the molecular arrangement of included fullerenes owing to their low symmetry cages. Thus, the various metastable molecular conformations of fullerenes in CPP are expected to 
observed depending on the experimental conditions.

Figure 4 shows the electronic energy level of the inclusion compounds of the large fullerene and [11]CPP together with that of an isolated fullerene and [11]CPP. We also show the squared wave functions of the highest occupied (HO) and the lowest unoccupied (LU) states. Since $\mathrm{C}_{76}\left(D_{2 d}\right)$ and $\mathrm{C}_{78}\left(C_{2 v}^{\prime}\right)$ fullerenes possess a deep LU state, the complexes exhibit interesting electronic structures near the Fermi level. The HO and LU states of the inclusion complexes exhibited staggered structures, where the HO and LU states were distributed on CPP and fullerene, respectively. According to the staggered electronic structure near the gap, the energy gap between $\mathrm{HO}$ and LU states (HO-LU gap) of the inclusion complexes was narrower than that of the isolated constituent unit. For the $[11] \mathrm{CPP} \supset \mathrm{C}_{76}$, the HO-LU gap was $0.903 \mathrm{eV}$, which is narrower than those of $\mathrm{C}_{76}$ and [11]CPP by 1.076 and $2.152 \mathrm{eV}$, respectively. For the [11]CPP $\supset \mathrm{C}_{78}$, the HO-LU gap was $0.688 \mathrm{eV}$, which is narrower than those of $\mathrm{C}_{78}$ and [11]CPP by 0.744 and $2.152 \mathrm{eV}$, respectively.

We further investigated the electronic structure modulation in terms of the inner spacing of CPP by changing the CPP species. Figure 5 shows the energy values of the electronic states associated with the fullerene and CPP around the HO-LU gap. The inclusion of fullerenes in CPP caused an upward shift of the HO and LU states of fullerenes. Furthermore, by decreasing the diameter of CPP, the HO and LU states monotonically shifted upward. The upward shift strongly depended on the spacing of CPP, so that the decrease of the CPP diameter caused a monotonic upward shift of the HO and LU states upon the inclusion. In contrast, the electronic states associated with the CPP exhibited different characteristics to those of the fullerenes. The LU states of [10]CPP and [11]CPP shifted downward upon inclusion of fullerene, while the LU state of [12]CPP and HO states of all CPP were insensitive to the inclusion.

Since the energy potential surfaces gradually decreased with approaching the center of the CPP, the electronic structure was also expected to be modulated upon the encapsulation process (Fig. 6). The HO and LU states of fullerene were sensitive to the fullerene position. The energies of the $\mathrm{HO}$ and LU states gradually decreased with separation from the center of CPP and asymptotically approached those of the isolated value at the fullerene position of $z=$ $8 \AA$ A. However, the HO and LU states of [11]CPP slightly shifted downward with approaching the center of [11]CPP. The downward shift of the states upon the inclusion was $0.1 \mathrm{eV}$. These facts indicated that the inner space of the CPP intrinsically caused the modulation of the electronic states of the included guest molecules, which depended on the spacing between fullerene and CPP. 
(a)

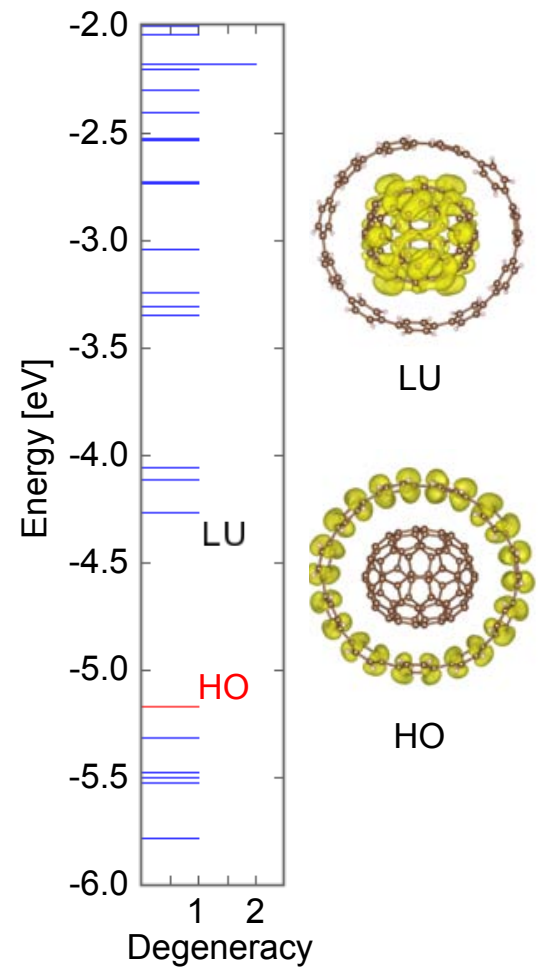

(c)

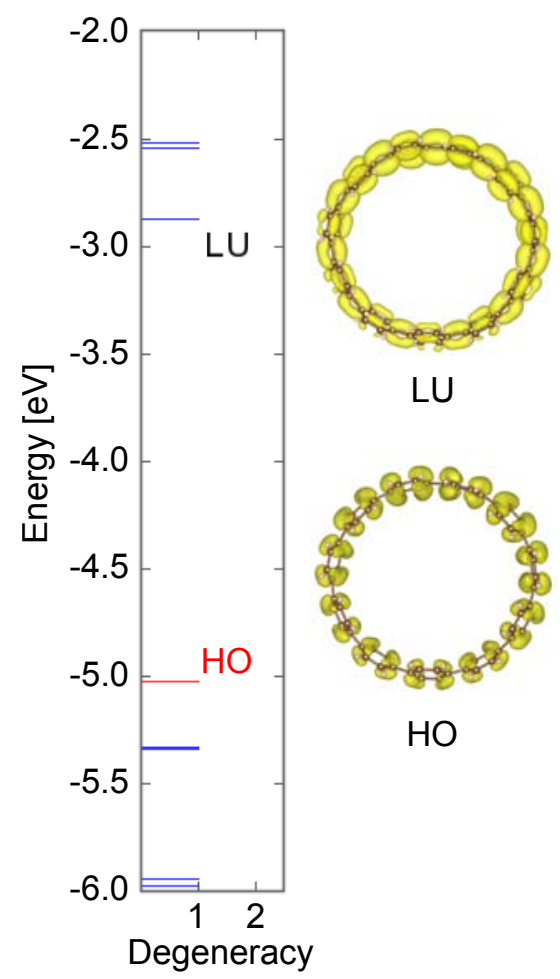

(b)

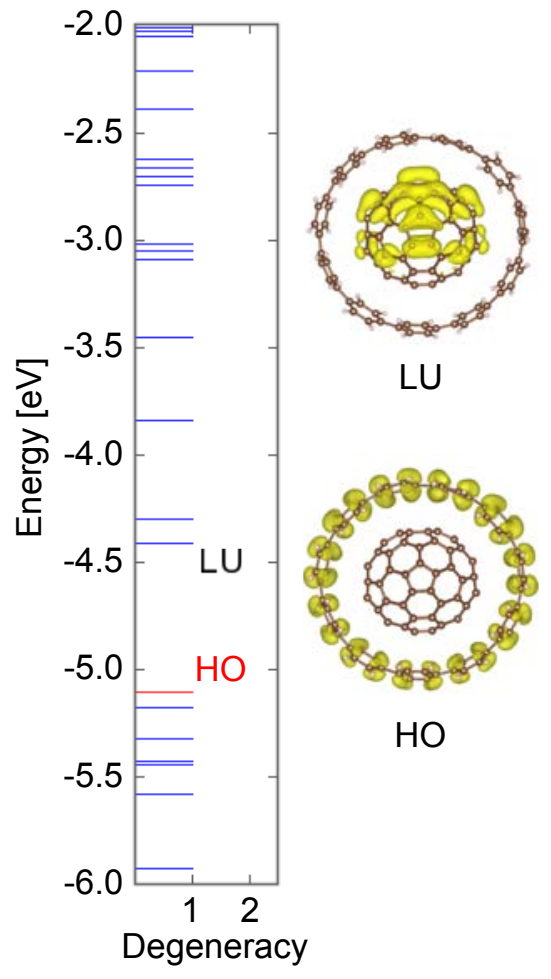

(d)

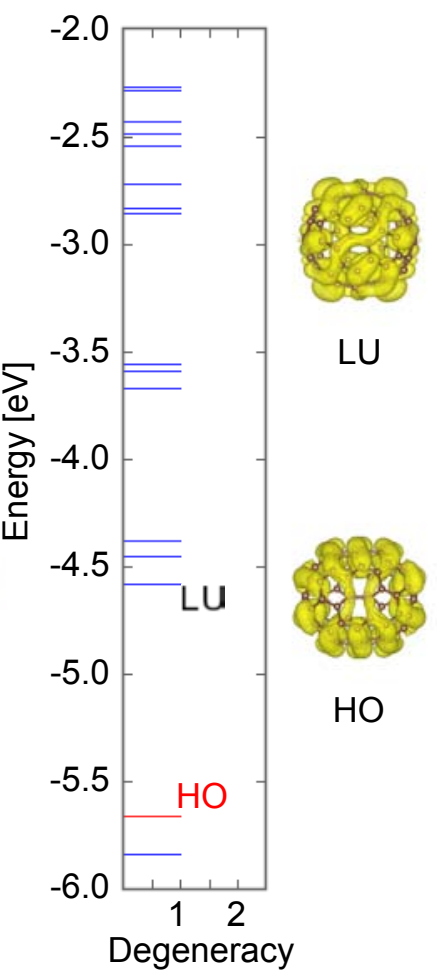

(e)

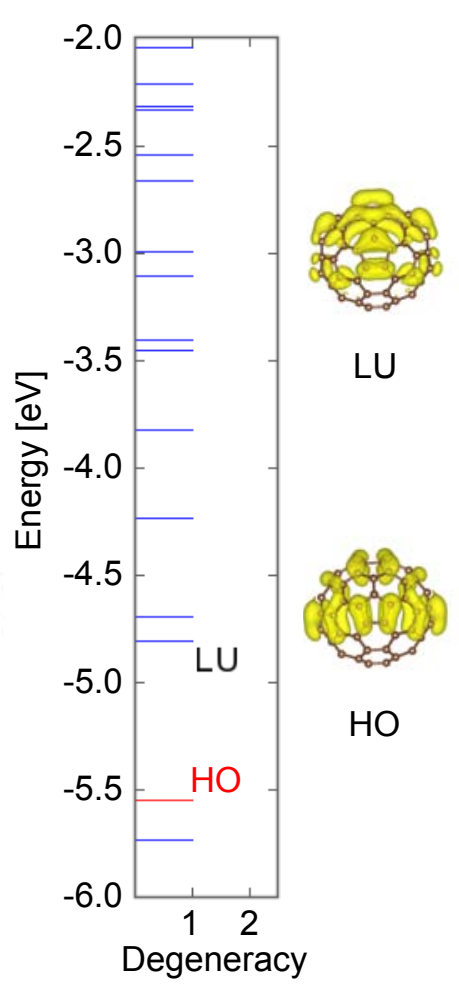

Fig. 4. The electronic energy level of $\mathrm{HO}$ and $\mathrm{LU}$ states of (a) $[11] \mathrm{CPP} \supset \mathrm{C}_{76}$, (b) $[11] \mathrm{CPP} \supset \mathrm{C}_{78}$, (c) [11]CPP, (d) $\mathrm{C}_{76}$, and (e) $\mathrm{C}_{78}$. 
(a)

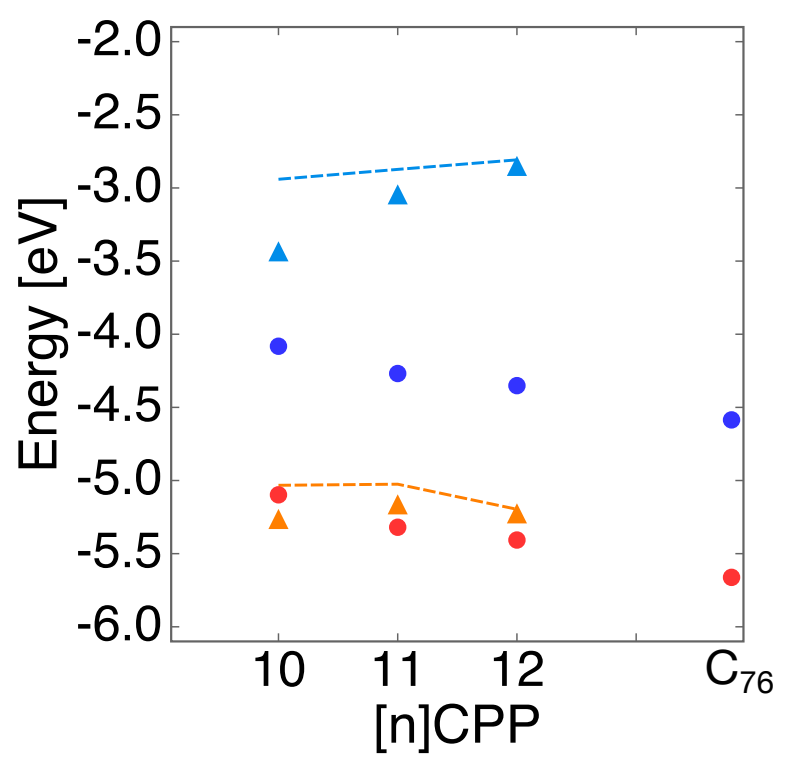

(b)

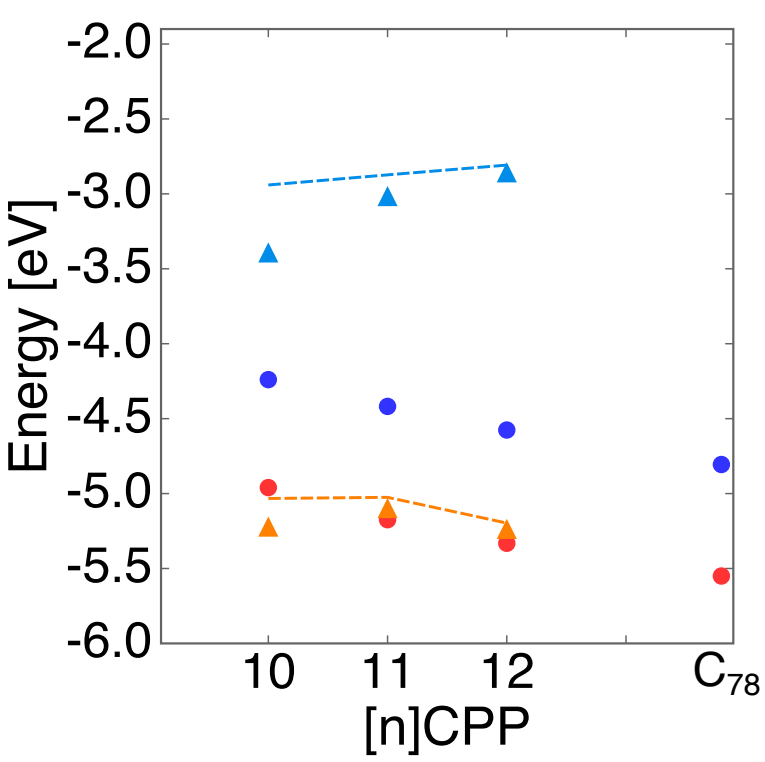

Fig. 5. The electronic energy levels associated with the HO and LU states of (a) $\mathrm{C}_{76}$ and [n]CPP in the inclusion complex and (b) $\mathrm{C}_{78}$ and [n]CPP in the inclusion complex. Circles, squares, triangles, inverted triangles, dashed line, and dotted line denote the HO of fullerenes in the complex, LU state of fullerenes in the complex, HO state of [n]CPP in the complex, LU state of [n]CPP in the complex, the HO state of the isolated [n]CPP, and LU state of the isolated [n]CPP, respectively. The HO and LU states of the isolated fullerenes are depicted on the right side of each panel.

To provide theoretical insight into the microscopic mechanism of the electronic structure modulation mentioned above, we investigated the modulation of the electrostatic potential $\Delta V$ upon fullerene inclusion into [11]CPP [Figs. 7(a) and 7(b)]. The potential modulation was evaluated by $\Delta V=V_{\text {comp }}-V_{\text {full }}-V_{\text {CPP }}$, where $V_{\text {comp }}, V_{\text {full }}$, and $V_{\text {CPP }}$ are the electrostatic potentials of inclusion complex, isolated fullerene, and [11]CPP, respectively. The electrostatic potential inside the [11]CPP exhibited an upward shift upon inclusion of the fullerene. This upward shift of the electrostatic potential was ascribed to the confinement effect on the electronic state of the fullerene included in [11]CPP. We also investigated charge redistribution upon the inclusion [Figs. 7(c) and 7(d)]. Upon the inclusion, the electron decreases at the vicinity of covalent network, while it increases in the space between fullerenes and [11]CPP. In addition to the vacuum spacing, the electron distribution also increases at the polar regions of included fullerenes. Thus the results imply that the inner space of the [11]CPP was insufficient to render the $\pi$ electron distribution of $\mathrm{C}_{76}$ and $\mathrm{C}_{78}$ fullerenes. 


\section{(a)}

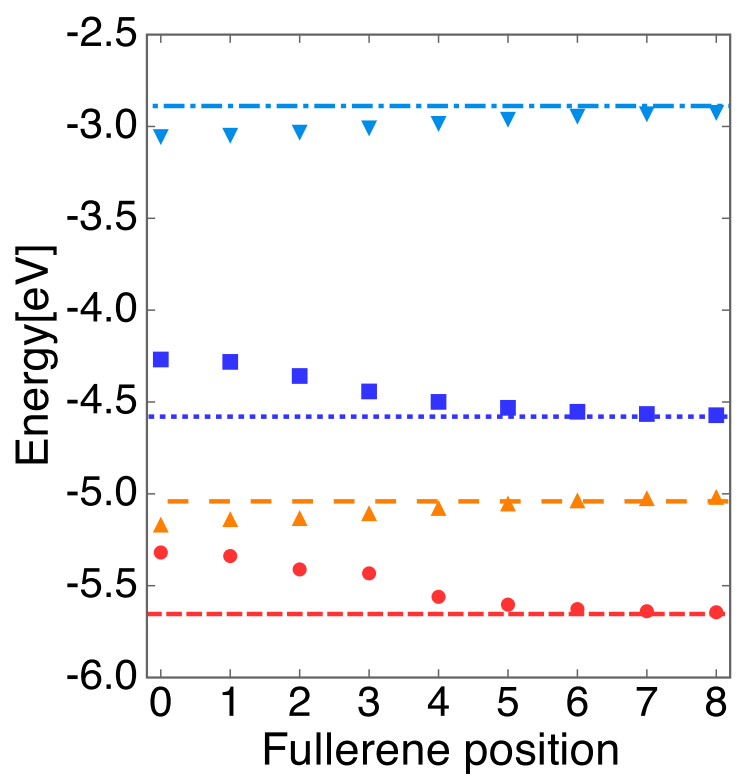

(b)

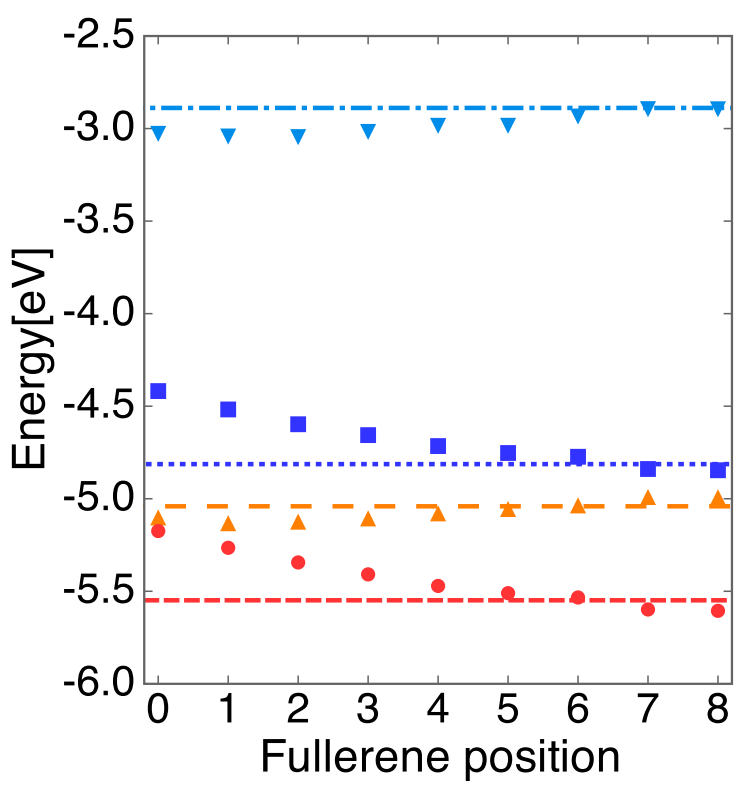

Fig. 6. The electronic energy levels associated with the HO and LU states of (a) $\mathrm{C}_{76}$ and [11]CPP in the form of [11]CPP $\supset \mathrm{C}_{76}$ and (b) $\mathrm{C}_{78}$ and [11]CPP in the form of [11]CPP $\supset \mathrm{C}_{78}$ as a function of the fullerene position along the molecular axis of [11]CPP. Circles, squares, triangles, inverted triangles, short dashed line, long dashed line, dotted-dashed line, and dotted line denote the HO of fullerenes in the complex, LU state of fullerenes in the complex, HO state of [11]CPP in the complex, LU state of [11]CPP in the complex, the HO state of the isolated fullerene, LU state of the isolated fullerene, the HO state of the isolated [11]CPP, and LU state of the isolated [11]CPP, respectively.

\section{Summary}

We studied the energetics and electronic structures of the carbon inclusion compounds, $[n] \mathrm{CPP} \supset \mathrm{C}_{76}$ and $[n] \mathrm{CPP} \supset \mathrm{C}_{78}$, where $n=10 \sim 12$, by first-principles total energy calculations based on density functional theory. Our calculations showed that the inclusion reactions of $\mathrm{C}_{76}$ and $\mathrm{C}_{78}$ fullerenes within $[n] \mathrm{CPP}$ were exothermic for $n \geq 11$, and were endothermic for $[10]$ CPP. The inclusion energy strongly depended on the diameter of the $[n]$ CPP. The largest inclusion energy was found to be $1.2 \mathrm{eV}$ per fullerene for [11]CPP, irrespective of the fullerene species studied here. The potential energy landscape along the inclusion reaction of fullerene did not possess any energy barrier, indicating that the fullerene molecule was spontaneously included within the inner space of [11]CPP. The electronic structure near the gap of these complexes exhibited interesting properties. The deep LU state of the fullerene molecule caused a staggered electronic structure near the HO-LU gap of the inclusion complexes. The 
$\mathrm{HO}$ and LU states were distributed on CPP and fullerene, respectively. We also found that the energy level associated with the fullerene was sensitive to the inner space of CPP. The energy levels monotonically increased with the decreasing diameter of CPP, in accordance with the quantum confinement effect by the CPP ring.

\section{Acknowledgment}

This work was supported by JST-CREST Grant Number JPMJCR1532 from the Japan Science and Technology Agency, JSPS KAKENHI Grant Number JP25246010, JP16H00898, and JP16H06331 from Japan Society for the Promotion of Science, and the Joint Research Program on Emission Energy Research, Institute of Advanced Energy, Kyoto University. Part of the calculations was performed on an NEC SX-Ace at the Cybermedia Center at Osaka University and on an SGI ICE XA/UV at the Institute of Solid State Physics, The University of Tokyo. 


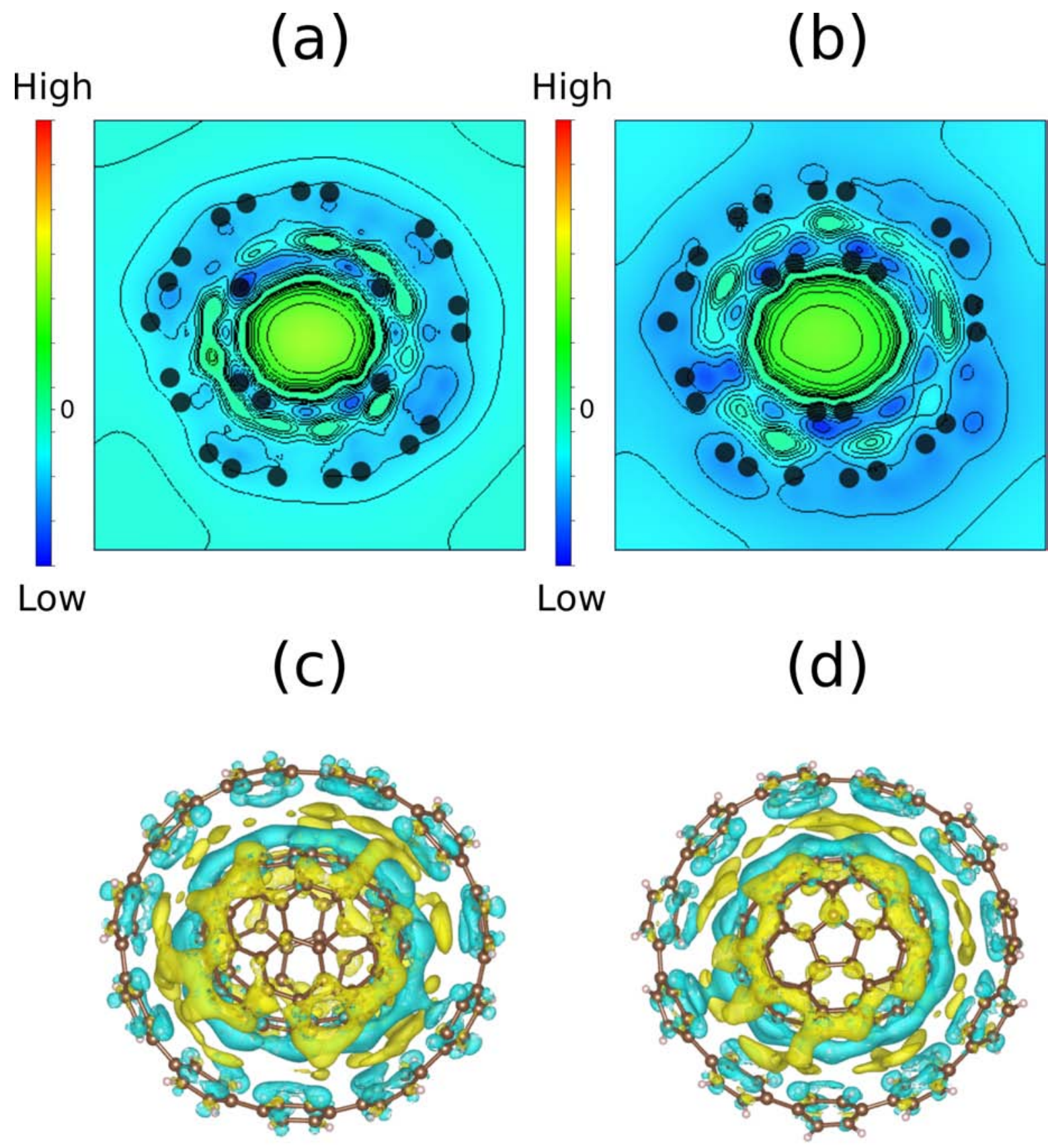

Fig. 7. Contour plot of the electrostatic potential modulation upon the inclusion of (a) $\mathrm{C}_{76}$ and (b) $\mathrm{C}_{78}$ in [11]CPP. Blue and yellow regions indicate the region where the potential energy decreases and increases, respectively, upon the inclusion. The charge redistribution upon the inclusion of (c) $\mathrm{C}_{76}$ and (d) $\mathrm{C}_{78}$ in [11]CPP. Blue and yellow regions indicate the region where the electrons decreases and increases, respectively, upon the fullerene inclusion. 


\section{References}

1) L. Salem: The Molecular Orbital Theory of Conjugated Systems (W. A. Benjamin, New York, NY, 1966).

2) M. J. S. Dewar: The Molecular Orbital Theory of Organic Chemistry (McGraw-Hill, New York, NY, 1969).

3) S. E. Stein and R. L. Brown, J. Am. Chem. Soc. 109, 3721 (1987).

4) G. S. Painter and D. E. Ellis, Phys. Rev. 1, 4747 (1970).

5) F. Bassani and G.P. Parravicini, Nuovo Cimento B 50, 95 (1967).

6) M. Posternak, A. Baldereschi, A. J. Freeman, E. Wimmer, and M. Weinert, Phys. Rev. Lett. 50, 761 (1983).

7) A.L. Mackay and H. Terrones, Nature 352, 762 (1991).

8) D. Vanderbilt and J. Tersoff, Phys. Rev. Lett. 68, 511, (1992).

9) G. E. Scuseria, Chem. Phys. Lett. 195, 534 (1992).

10) H. Terrones and A. L. MacKay, Carbon 30, 1251 (1992).

11) T. Lenosky, X. Gonze, M. Teter, and V. Elser, Nature 355, 333 (1992).

12) S. Iijima, Nature 354, 56 (1991).

13) N. Hamada, S. Sawada, and A. Oshiyama, Phys. Rev. Lett. 68, 1579 (1992).

14) R. Saito, M. Fujita, M. S. Dresselhaus, and G. Dresselhaus, Appl. Phys. Lett. 60, 2204 (1992).

15) K. Tanaka, K. Okahara, M. Okada, and T. Yamabe, Chem. Phys. Lett. 191, 469 (1992).

16) H.W. Kroto, J.R. Heath, S.C. O’Brien, R.F. Curl, and R.E. Smalley, Nature 318, 162 (1985).

17) P.W. Fowler and D.E. Manolopoulos: An Atlas of Fullerenes (Oxford University Press, Oxford, U. K., 1995).

18) D.E. Manolopoulos and P.W. Fowler, J. Chem. Phys. 96, 7603 (1992).

19) B.L. Zhang, C.Z. Wang, and K.M. Ho, Chem. Phys. Lett, 193, 225 (1992).

20) B.L. Zhang, C.Z. Wang, K. M. Ho, C.H. Xu, and C.T. Chan, J. Chem. Phys. 98, 3095 (1993).

21) S. Saito, S. Okada, S. Sawada, and N. Hamada, Phys. Rev. Lett. 75, 685 (1995).

22) S. Okada and S. Saito, Chem. Phys. Lett. 252, 94 (1996). 
23) W. Krätschmer, L.D. Lamb, K. Fostiropoulous, and D.R. Hoffman, Nature 347, 354 (1990).

24) S. Saito and A. Oshiyama, Phys. Rev. Lett. 66, 2637 (1991).

25) S. Okada and S. Saito, Phys. Rev. B 59, 1930 (1998).

26) S. Okada, S. Saito, and A. Oshiyama, Phys. Rev. Lett. 83, 1986 (1999).

27) A. Thess, R. Lee, P. Nikolaev, H. Dai, P. Petit, J. Robert, C. Xu, Y. H. Lee, S. G. Kim, A.G. Rinzler, D. T. Colbert, G. E. Scuseria, D. Tománek, J. E. Fischer, and R.E. Smalley, Science 273, 483 (1996).

28) J.-C. Charlier, X. Gonze, and J.-P. Michenaud, Europhys. Lett. 29, 43 (1995).

29) P. Delaney, H. J. Choi, J. Ihm, S. G. Louie, and M. L. Cohen, Nature 391, 466 (1998).

30) B. W. Smith, M. Monthioux, and D. E. Luzzi, Nature 396, 323 (1998).

31) B. Burteaux, A. Claye, B.W. Smith, M. Monthioux, D. E. Luzzi, and J. E. Fischer, Chem. Phys. Lett. 310, 21 (1999).

32) B. W. Smith, M. Monthioux, and D. E. Luzzi, Chem. Phys. Lett. 315, 31 (1999).

33) S. Okada, S. Saito, and A. Oshiyama, Phys. Rev. Lett., 86, 3835 (2001).

34) M. Otani, S. Okada, and A. Oshiyama, Phys. Rev. B 68, 125424 (2003).

35) T. Iwamoto, Y. Watanabe, T. Sadahiro, T. Haino, and S. Yamago, Angew. Chem. Int. Ed. 50, 8342 (2011).

36) H. Isobe, S. Hitosugi, T. Yamasaki, and R. Iizuka, Chem. Sci. 4, 1293 (2013).

37) T. Iwamoto, Y. Watanabe, H. Takaya, T. Haino, N. Yasuda, and S. Yamago, Chem. Eur. J. 19, 14061 (2013).

38) R. Ettl, I. Chao, F. Diederich, and R.L. Whetten, Nature 353, 149 (1991).

39) F. Diederich, R.L. Whetten, C. Thulgen, R. Ettle, I. Chao, and M. Alvarez, Science 254, 1768 (1991).

40) K. Kikuchi, N. Nakahara, T. Wakabayashi, S. Suzuki, H. Shiromaru, Y. Miyake, K. Saito, I. Ikemoto, M. Kainosho, and Y. Achiba, Nature 357, 142 (1992).

41) P. Hohenberg and W. Kohn, Phys. Rev. 136, B864 (1964).

42) W. Kohn and L.J. Sham, Phys. Rev. 140, A1133 (1965).

43) Y. Morikawa, K. Iwata, and K. Terakura, Appl. Surf. Sci. 169-170, 11 (2001).

44) J.P. Perdew and A. Zunger, Phys. Rev. B 23, 5048 (1981).

45) D.M. Ceperley and B.J. Alder, Phys. Rev. Lett. 45, 566 (1980).

46) D. Vanderbilt, Phys. Rev. B 41, 7892 (1990). 